Article

\title{
Quality Control of the Continuous Hot Pressing Process of Medium Density Fiberboard Using Fuzzy Failure Mode and Effects Analysis
}

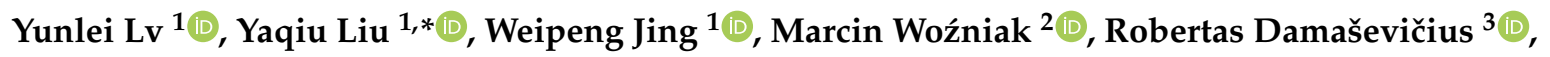 \\ Rafał Scherer ${ }^{4}(1)$ and Wei Wei ${ }^{5,6}$ (i) \\ 1 College of Information and Computer Engineering, Northeast Forestry University, Harbin 150040, China; \\ yunleilv@nefu.edu.cn (Y.L.); jwp@nefu.edu.cn (W.J.) \\ 2 Faculty of Applied Mathematics, Silesian University of Technology, 44-100 Gliwice, Poland; \\ marcin.wozniak@polsl.pl \\ 3 Department of Applied Informatics, Vytautas Magnus University, 44404 Kaunas, Lithuania; \\ robertas.damasevicius@vdu.lt \\ 4 Department of Intelligent Computer Systems, Częstochowa University of Technology, 42-200 Częstochowa, \\ Poland; rafal.scherer@pcz.pl \\ 5 School of Computer Science and Engineering, Xi'an University of Technology, Xi'an 710048, China; \\ weiwei@xaut.edu.cn \\ 6 Shaanxi Key Laboratory for Network Computing and Security Technology, Xi'an 710054, China \\ * Correspondence: yaqiuliu@nefu.edu.cn
}

Received: 13 June 2020; Accepted: 30 June 2020; Published: 3 July 2020

\begin{abstract}
In this paper, a fuzzy failure mode and effects analysis (FMEA) method is proposed by combining fault theory and a failure analysis method. The method addresses the problem of board thickness control failure and the problem of thickness deviation defect blanking, which can occur during continuous hot pressing (CHP) process, which is one of the most important processes in the production of medium-density fiberboard (MDF). The method combines the fault analysis with the Hamming code method and using the Hamming code to calculate and represent the cylinder array of the continuous hot-pressed thickness control execution unit to analyze and process the potential fixed thickness failure modes in MDF hot press production, and then summarizes the decision rules for controlling the board thickness and the level of sheet deviation. By combining the fuzzy FMEA method of the Hamming code and the logical OR operation of the experimental analysis, the method of thickness deviation and recognition control fault information for the CHP of MDF, which is proposed in this paper, permits the increase of the number of error levels, which makes optimization for controller more convenient and improves the efficiency to recognize errors.
\end{abstract}

Keywords: MDF; fuzzy FMEA; continuous hot pressure; deviation recognition

\section{Introduction}

Medium-density fiberboard (MDF) is the second most popular (12.3 million $\mathrm{m}^{3}$ in 2017) wood-based composites in the manufacture of furniture and interior decoration in Europe [1], and is an excellent alternative to a range of natural wood panels due to the excellent mechanical properties and desired physical features. In the production of MDFs, wood fiber and glue at $640-800 \mathrm{~kg} / \mathrm{cm}^{3}$ density are used [2]. The production of MDF involves the application of hot pressing using various heat processing temperatures $\left(155^{\circ} \mathrm{C}, 165^{\circ} \mathrm{C}\right.$, and $\left.175^{\circ} \mathrm{C}\right)$ for different time durations $(2.5 \mathrm{~h}, 3.5 \mathrm{~h}$, and $4.5 \mathrm{~h})$ [2]. With the introduction of the continuous hot-pressing (CHP) technology in Germany in the beginning of the 1970s, productivity was increased, and good surface qualities 
were achieved [3]. Hot pressing is the most crucial process in the production of MDF [4], and it is essential to achieve the required quality of MDF. CHP is a process wherein the raw wood board is subjected to the application of temperature and pressure in order to achieve moisture evaporation, while in the process, the density increases, the glue solidifies, and the raw material undergoes physical and chemical changes. As a result, the bonding force between fibers is formed [5]. The MDF slab is pressed to a required thickness under pressure. The resulting quality of the MDF depends on the slab formation. Due to steam pressure during CHP, thickness deviations can arise as in thicker MDF slab areas, the steam cannot be released under the constant pressure [6]. Thickness is the principle element of achieving high-quality MDF. As a result, multiple research efforts are done to solve the thickness control problem for MDF CHP systems [7-9].

A continuous flat press is the core equipment of the entire MDF production line [10]. Quality control is an essential part of the manufacturing process [11,12]. The production capacity and product quality of the entire MDF production line are directly affected by the accuracy and reliability of its operation and control. The needs to support the requirements of scale and manufacturing intensification, as well as to maintain the quality control of the board thickness deviation of the continuous flat pressing (CFP) process and to ensure the continuous and reliable operation of the press have become a core issue of the MDF manufacturing industry. The MDF hot press forming process is to compact the fluffy mat of fibers by applying pressure; at the same time, the adhesive is solidified, and a compaction and adhesion point is formed between the fiberboard by heating, thereby forming a certain thickness [5].

FMEA is a reliability management method generally employed in different industrial applications to assure the security and reliability of systems and services [13]. FMEA is more arbitrary for the analysis of the level of the failure mode of MDF. The result is not very accurate [14]. This will cause the failure mode analyzed to be too one-sided and difficult to correct [8].

To overcome these problems, this paper proposes to fuse the original FMEA method through the theory of fuzzy mathematics and obtain a new fuzzy FMEA method to judge the deviation grade of MDF accurately. It relies on consistent judgment of the deviation pattern of the sheet surface. The data used in this paper are sampled data from laboratory sensors. The FMEA system is analyzed by fuzzy mathematics and the fuzzy matrix method, and different FRPN values are calculated. FRPN is the fuzzy risk priority number according to the FMEA's judgment standard; the RPN is the only criterion for judging the FMEA failure mode level [9]. In view of the above problems, this paper starts with fault diagnosis control and uses the failure mode and impact analysis (FMEA) to analyze theoretically the possible failure mode of the continuous flat press. Through the FRPN determination of the fuzzy FMEA method, the deviation degree of the continuous hot pressing process of the medium-density fiberboard is more accurately determined, and the specific deviation mode is compared with the sensor sampling data calculated by the Hamming coding, while the medium-density fiberboard is compared with the verification method. The consistency judgment of the deviation level is made.

Therefore, this paper proposes to use fuzzy FMEA method to identify and diagnose the deviation of continuous heat pressing of MDF. Our contributions are summarized as follows:

(1) The deviation grade information of continuous heat pressing of medium-density fiberboard analyzed by the fuzzy FMEA method is more accurate than the original FMEA method. For comparison, the decision matrix logic OR operation result of the Hamming coding analysis is used to verify the proposed method.

(2) Through the fuzzy FMEA method used to analyze the deviation level information, the solution can be found more quickly and the problem can be corrected and rectified in time. 


\section{Methodology}

\subsection{Related Work}

Primarily, an FMEA uses ordinal subjective scores to capture risk information. One type of proactive risk assessment technique is failure modes and effects analysis (FMEA). The area of Fuzzy FMEA research has become more and more extensive in recent years. Nie et al. [15] presented a quantitative risk analyzing method that integrated the interval type-2 fuzzy sets (IT2FSs) with FMEA. Wu et al. [16] stated that while the FMEA can find possible failures and calculate the risk priority number (RPN), the IT2FSs can deal better with the ambiguity and vagueness of the linguistic assessment of experts. Qin et al. [17] combined IT2FSs with the evidential reasoning (ER) method in order to deal with uncertainties more efficiently. An event of an oil spill from a ship was used to showcase the applicability of the suggested method with regard to the damage to the marine environment [18]. In [19], fuzzy-grey correlation analysis was employed for safety risk assessment of a mine to prevent industrial mining accidents. A similar solution was adopted in [20] for controlling cyber-physical-systems (CPS) in coal mine tunnels. Seiti et al. [21] extended a pessimistic-optimistic model of fuzzy belief structure (FBS) for fuzzy evidential FMEA problems. Aiming to evaluate the proposed method, they applied it to the fuzzy and crisp Dempster-Shafer theory (DST) structure. Liu et al. [22] studied an FMEA process for fishing vessels as a case study, and the results were compared with the risk-free mode. McElroy et al. [23] used FMEA for detailed process mapping, identification, and ranking of possible failure by adopting the risk probability number (RPN). Fattahi and Khalilzadeh [24] modified FMEA by replacing RPN with fuzzy-weighted RPN (FWRPN), and after computing FWRPN for each failure, the identified failures were eliminated or corrected. Li and Chen [25] extended FMEA by integrating FBS and the grey relational projection method (GRPM) to replace RPN, thus overcoming its limitations. Shafiee et al. [26] suggested a combined FTAand FMEA model for performing risk analysis of safety-critical systems, which used the weights based on Birnbaum's measure of importance to revise RPNs. Fang et al. [27] proposed to combine variable precision rough numbers and the prospect theory as a new method for FMEA that was capable of dealing with vague information. Wu et al. [16] used the Hamming tree to analyze the obtained code bits. Firstly, different fault modes were analyzed, then the deviation level threshold was defined, and the deviation level was divided. Secondly, the collaborative board thickness correction diagnosis method was established, and the control fault information expression and decision mapping were performed. Chen and Deng [28] extended the FMEA model with Dempster-Shafer (DS) evidence theory and the grey relational projection approach.

Here, we employ the FMEA technique to improve the deviation of MDF continuous hot pressing. To achieve this aim, we propose a failure mode and effect analysis (FMEA)-based method for the deviation and identification of MDF continuous hot pressing.

\subsection{MDF Processing Mechanism}

The processing mechanism of MDF is usually divided into three parts according to the length of the press: the fast closing part, the spacing holding part, and the constant thickness part, as shown in Figure 1. Depending on the slab material, slab thickness, and density requirements, the spacing of the hot board has different settings [29]. The combination of the pitch setting curve and the actual pitch curve reflects the pressure of the slab in the press. In the interval maintaining portion, the thickness of the slab is highly variable, so it is necessary to adjust the spacing of the hot pressing plates near the exit of the hot press, thereby setting the thickness of the slab and stabilizing its thickness [30]. Usually, considering the rebound characteristics of the slab, the pitch setting of the fixed thickness section should be slightly smaller than the set slab thickness value.

Through the above MDF type mechanism analysis, it can be known that the continuous flat hot pressing system has a complicated process control mechanism, which brings difficulty for accurate control of the slab thickness, especially when there is a deviation between the real thickness and the 
set thickness of the slab. Note that the dimensions of the MDF board are determined using the ISO 16895:2016 standard. The slab thickness correction needs to be achieved through a reasonable and effective control mechanism. The control of slab thickness is an important part and is always related to the final quality of the production [31]. The failure mode of the fixed thickness section is also an important part of the deviation control of the MDF fixed thickness section. The impact of different failure modes on the quality of medium-density fiberboard products and the high yield pass rate are predicted in advance. By analyzing the failure mode of the fixed thickness section, the corresponding code position can be obtained, and the principle of the Hamming code is used to control the deviation level. For the deviation control of MDF, this study integrates the FMEA failure mode expression into the Hamming tree coding analysis method to achieve the MDF fixed thickness deviation control.

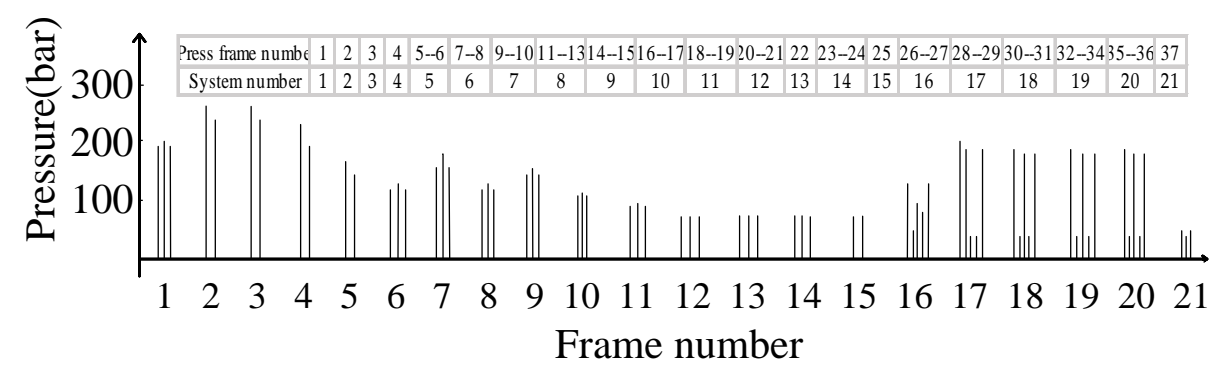

Figure 1. Medium-density fiberboard (MDF) hot pressing pressure trend curve.

The hot pressing mechanism shown in Figure 1 takes 37 sets of cylinder arrays as an example and analyzes the hot pressing pressure changes in three different regions and states. From the trend in the figure, it can be seen that Groups 1-6 are a low pressure area, and after 6 groups, the pressure is flat as a pressure holding area, while the pressure needs to be increased because the board needs to be given a thickness when entering the high pressure area.

\subsection{FMEA of Continuous Hot Pressing}

FMEA is one of the common methods for the reliability analysis and preventive design of products [32]. It aims to identify possible failures in the product design process and equipment design phase and then analyze the failure modes of each component to find a way to influence the previous level and even the system. FMEA analysis is a program that analyzes the cause of the failure by analyzing all possible failure modes of the system to determine the impact of potential failure modes on personnel safety, system functions, maintenance measures, etc., which is a positive preventive approach [33]. FMEA is a quality control method that reflects the concept of prior prevention and control. The application worldwide shows that most of the quality defects can be solved by applying the FMEA analysis method, helping the enterprise to reduce the risk of the potential failure of the product effectively, thereby scientifically and systematically solving the quality problem and helping the enterprise to improve the production quality.

FMEA is one of the analytical methods used for safety evaluation. Its basic principle is to divide the system into components or elements, then analyze the potential failure modes of the equipment components, the factors of the failure, and the consequences, and finally, develop corresponding control measures based on the evaluation results. FMEA divides the system or device into functional failure levels, reasonably summarizes the potential failure modes of the entire system or equipment organizational units, and ranks the risk according to the severity of the consequences of the failure to determine preventive compensation measures for the system or equipment and improve the reliability of the system or equipment [15]. The risk priority number (RPN) is calculated by evaluating the equipment failure severity (S), the degree of occurrence (O), and the degree of detection (D). RPN is the product of severity $(\mathrm{S})$, the degree of occurrence $(\mathrm{O})$, and degree of detection $(\mathrm{D})$. It is a way to evaluate risk. The size of RPN can indicate the magnitude of the risk [34]. The severity (S) indicates the 
effect of the failure mode on the consequences; the degree of occurrence $(\mathrm{O})$ indicates the probability of the occurrence of the failure; and the degree of detection (D) indicates the difficulty of detecting the failure mode. The scoring criteria for $\mathrm{O}, \mathrm{S}$, and $\mathrm{D}$ are shown in Table 1.

Table 1. Three parameters O, S, and D scoring criteria of Fuzzy Failure Mode and Effects Analysis (FMEA).

\begin{tabular}{llll}
\hline Occurrence (O) & Severity (S) & Detection (D) & Rating \\
\hline Faults rarely occur & $\begin{array}{l}\text { Normal, only weakly affecting } \\
\text { certain components }\end{array}$ & $\begin{array}{l}\text { The probability of failure to } \\
\text { detect is low }\end{array}$ & 2,3 \\
\hline Occasionally, the device can run & The overall performance is reduced & Occasionally, it is not detected & $4,5,6$ \\
\hline Failure occurs often & $\begin{array}{l}\text { The function of the device is } \\
\text { basically lost }\end{array}$ & $\begin{array}{l}\text { The probability of failure to } \\
\text { operate is high }\end{array}$ & 7,8 \\
\hline Failure occurs inevitably & Equipment damage & $\begin{array}{l}\text { The probability of not being } \\
\text { detected is extremely high }\end{array}$ & 9,10 \\
\hline
\end{tabular}

The core factor of FMEA is RPN, which is the only evaluation standard for determining the failure mode. The calculation formula of RPN is:

$$
R P N=S \cdot O \cdot D
$$

In mathematical terms, points can be lines, and lines can be surfaces. Therefore, five points can determine a line through which we can determine its trend. In this way, the range of deviation grades of MDF can be preliminarily determined in this way. The results of the preliminary judgment are four types of failures: two types of slopes, depressions, and bulges. The system analyzes the failure mode and influence of MDF continuous hot pressing [35-37]. It is assumed that the operating state of the press is normal, and there is no fault. We analyze the influence of different control modes on the pressure cylinder. The defects and deviations of the plates are analyzed as follows: At present, only the fixed thickness section is studied, and the error state of the sensor itself is ignored to analyze the failure mode of the MDF continuous hot pressing section. There are four categories of defects for the board: slopes (two types of slopes on the left and right slopes), depressions, and bulges. The four types of failure modes are analyzed and decided. Each type of deviation is divided into five types. The basis for the division of A, B, C, D, and E is based on the deviation of the thickness of the MDF continuous hot pressing section. The classification of the light, medium, and heavy grades is based on the sheet defects and failure modes of the sheet surface. A, B, C, D, and E represent different grades of the same failure mode, which are light, moderate, and severe, and the light refinement is divided into $A$ and $B$, while the severe is divided into $C$ and $D$. The basis of classification is the judgment criteria presented in Table 1. The surface defects of the MDF continuous hot board of the FMEA's mode are shown in Figure 2. 

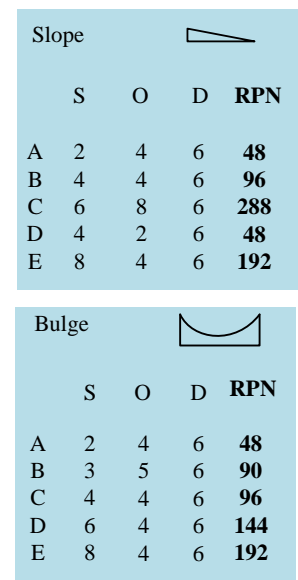
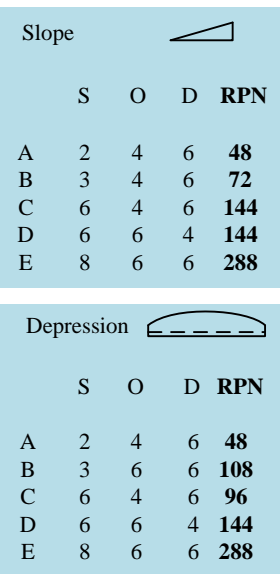

Figure 2. MDF's FMEA grade analysis chart.

In this paper, five sensors will be used as the pressure matrix array combined output matrix mode, and the cylindrical array is shown in Figure 3. The Hamming code can detect up to two bits of error or correct a bit error without detecting an uncorrected error by adding a check digit to the data bit. According to the failure mode analysis of the above-mentioned MDF continuous hot pressing FMEA table, the four kinds of deviation levels obtained by the FMEA analysis are designed as follows, and each matrix corresponds to one failure mode. In the matrix, 0 represents the pressure output, and 1 represents the displacement output. The matrix contains the level of deviation and the sensors. The data generated in this article are sample data from five sensors of the laboratory's Grecon equipment. The Hamming code of MDF deviation information is obtained by sampling data of the sensor, as shown in Table 2. The number 0 represents pressure control, and the number 1 represents displacement control. By adjusting the control mode of 6 groups of cylinders, the effect of correcting the deviation is achieved. The matrix is the level of the MDF continuous hot pressing level.

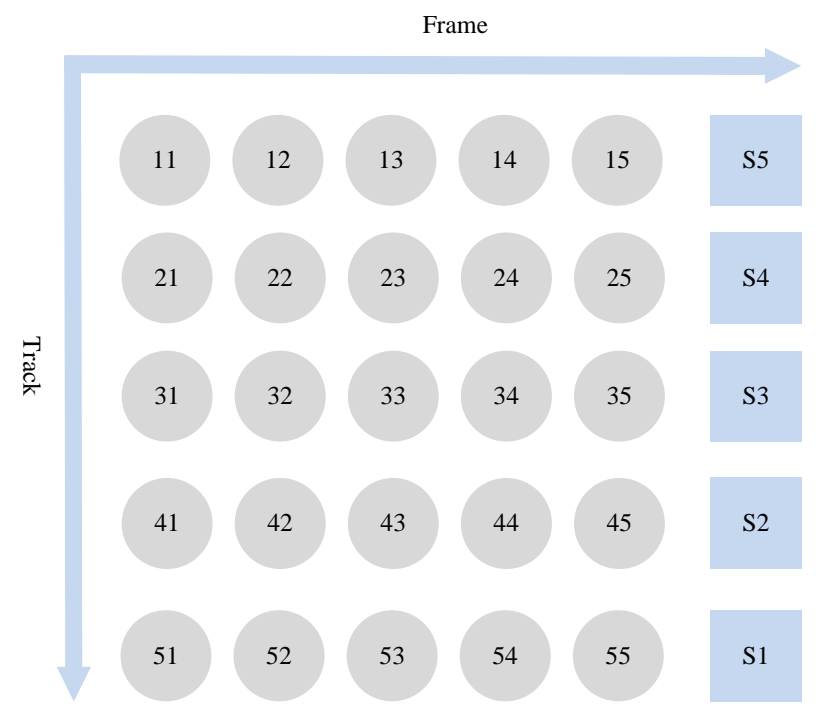

Figure 3. Cylinder array combined output matrix mode.

$$
M_{0, S}=\left[\begin{array}{lllll}
0 & 1 & 0 & 0 & 0 \\
0 & 1 & 0 & 0 & 0 \\
0 & 1 & 0 & 0 & 0 \\
0 & 1 & 0 & 0 & 0 \\
0 & 1 & 0 & 0 & 0
\end{array}\right] M_{A, 1}=\left[\begin{array}{lllll}
0 & 1 & 0 & 0 & 0 \\
0 & 1 & 1 & 0 & 0 \\
0 & 1 & 0 & 0 & 0 \\
0 & 1 & 0 & 0 & 0 \\
0 & 1 & 0 & 0 & 0
\end{array}\right]
$$




$$
\begin{aligned}
& M_{A, 2}=\left[\begin{array}{lllll}
0 & 1 & 0 & 0 & 0 \\
0 & 1 & 0 & 0 & 0 \\
1 & 1 & 1 & 0 & 0 \\
0 & 1 & 0 & 0 & 0 \\
0 & 1 & 0 & 0 & 0
\end{array}\right] M_{A, 3}=\left[\begin{array}{lllll}
0 & 1 & 0 & 0 & 0 \\
0 & 1 & 1 & 0 & 0 \\
0 & 1 & 1 & 0 & 0 \\
0 & 1 & 0 & 0 & 0 \\
0 & 0 & 0 & 0 & 0
\end{array}\right] \\
& M_{A, 4}=\left[\begin{array}{lllll}
0 & 1 & 1 & 0 & 0 \\
0 & 1 & 0 & 0 & 0 \\
0 & 1 & 1 & 0 & 0 \\
0 & 1 & 0 & 0 & 0 \\
0 & 0 & 0 & 0 & 0
\end{array}\right] M_{A, 5}=\left[\begin{array}{lllll}
0 & 1 & 0 & 0 & 0 \\
0 & 1 & 0 & 0 & 0 \\
0 & 1 & 1 & 0 & 0 \\
0 & 1 & 1 & 0 & 0 \\
0 & 0 & 0 & 0 & 0
\end{array}\right] \\
& M_{B, 1}=\left[\begin{array}{lllll}
0 & 1 & 0 & 0 & 0 \\
0 & 1 & 0 & 0 & 0 \\
1 & 1 & 1 & 0 & 0 \\
0 & 1 & 0 & 0 & 0 \\
0 & 1 & 0 & 0 & 0
\end{array}\right] M_{B, 2}=\left[\begin{array}{lllll}
0 & 1 & 0 & 0 & 0 \\
0 & 1 & 0 & 0 & 0 \\
1 & 1 & 1 & 0 & 0 \\
0 & 1 & 0 & 0 & 0 \\
0 & 1 & 0 & 0 & 0
\end{array}\right] \\
& M_{B, 3}=\left[\begin{array}{lllll}
0 & 1 & 0 & 0 & 0 \\
0 & 1 & 0 & 0 & 0 \\
1 & 1 & 1 & 0 & 0 \\
0 & 1 & 0 & 0 & 0 \\
0 & 1 & 0 & 0 & 0
\end{array}\right] M_{B, 4}=\left[\begin{array}{lllll}
0 & 1 & 0 & 0 & 0 \\
0 & 1 & 0 & 0 & 0 \\
1 & 1 & 1 & 0 & 0 \\
0 & 1 & 0 & 0 & 0 \\
0 & 1 & 0 & 0 & 0
\end{array}\right] \\
& M_{B, 5}=\left[\begin{array}{lllll}
0 & 1 & 0 & 0 & 0 \\
0 & 1 & 0 & 0 & 0 \\
1 & 1 & 1 & 0 & 0 \\
0 & 1 & 0 & 0 & 0 \\
0 & 1 & 0 & 0 & 0
\end{array}\right] M_{C, 1}=\left[\begin{array}{lllll}
0 & 0 & 0 & 0 & 0 \\
0 & 1 & 0 & 0 & 0 \\
0 & 1 & 0 & 0 & 0 \\
1 & 1 & 0 & 0 & 0 \\
1 & 1 & 0 & 0 & 0
\end{array}\right] \\
& M_{C, 2}=\left[\begin{array}{lllll}
0 & 0 & 0 & 0 & 0 \\
0 & 1 & 0 & 0 & 0 \\
0 & 1 & 0 & 0 & 0 \\
1 & 1 & 1 & 0 & 0 \\
1 & 1 & 0 & 0 & 0
\end{array}\right] M_{C, 3}=\left[\begin{array}{lllll}
0 & 0 & 0 & 0 & 0 \\
0 & 1 & 0 & 0 & 0 \\
1 & 1 & 1 & 0 & 1 \\
0 & 1 & 1 & 0 & 0 \\
0 & 0 & 0 & 0 & 0
\end{array}\right] \\
& M_{C, 4}=\left[\begin{array}{lllll}
0 & 0 & 0 & 0 & 0 \\
0 & 1 & 0 & 0 & 0 \\
1 & 1 & 1 & 0 & 1 \\
0 & 1 & 1 & 0 & 0 \\
0 & 1 & 0 & 0 & 0
\end{array}\right] M_{C, 5}=\left[\begin{array}{lllll}
1 & 1 & 0 & 0 & 0 \\
1 & 1 & 0 & 0 & 0 \\
1 & 1 & 0 & 0 & 0 \\
0 & 1 & 0 & 0 & 0 \\
1 & 0 & 1 & 0 & 0
\end{array}\right] \\
& M_{E, 1}=\left[\begin{array}{lllll}
0 & 0 & 0 & 0 & 0 \\
0 & 1 & 0 & 0 & 0 \\
0 & 1 & 0 & 0 & 1 \\
1 & 1 & 1 & 0 & 1 \\
1 & 1 & 1 & 0 & 1
\end{array}\right] M_{E, 2}=\left[\begin{array}{lllll}
0 & 0 & 0 & 0 & 0 \\
0 & 1 & 0 & 0 & 0 \\
0 & 1 & 0 & 1 & 1 \\
1 & 1 & 1 & 0 & 1 \\
1 & 1 & 1 & 0 & 1
\end{array}\right] \\
& M_{E, 3}=\left[\begin{array}{lllll}
0 & 0 & 0 & 0 & 0 \\
1 & 1 & 1 & 0 & 1 \\
1 & 1 & 1 & 0 & 1 \\
1 & 1 & 1 & 1 & 1 \\
0 & 0 & 0 & 0 & 0
\end{array}\right] M_{E, 4}=\left[\begin{array}{lllll}
0 & 0 & 0 & 0 & 0 \\
0 & 1 & 0 & 0 & 1 \\
0 & 1 & 0 & 0 & 1 \\
1 & 1 & 1 & 0 & 1 \\
1 & 1 & 1 & 0 & 1
\end{array}\right] \\
& M_{E, 5}=\left[\begin{array}{ccccc}
1 & 1 & 1 & 0 & 1 \\
1 & 1 & 1 & 0 & 1 \\
0 & 1 & 0 & 0 & 0 \\
0 & 1 & 0 & 0 & 1 \\
0 & 0 & 0 & 0 & 1
\end{array}\right]
\end{aligned}
$$


The final decision matrix is calculated by Equation (2), where $X$ represents the actual error level and $s$ represent the sensor's index.

$$
M_{\text {final }}=\operatorname{or}\left(M_{X, s}\right)_{X=[A, E] ; s=1,2,3,4,5}
$$

\subsection{Fuzzy FMEA-Based MDF Continuous Pressing}

This paper refers to the method of fuzzy calculation in [22]. This method is used to solve the evaluation of the shipboard integrated power system. It is similar to the method used in this paper to solve the problem of the quality control of the CHP process of MDF. This method can be applied to solve similar problems. Therefore, this article cites this method for the research in this article. The equations for applying the article's method are listed below:

$$
\left\{\begin{array}{l}
r_{j}=\sum_{k=1}^{3} r_{j k} \\
\pi_{j k}=r_{j k} / r_{j} \\
H\left(r_{j}\right)=-\sum_{k=1}^{3} \pi_{j k} \ln \pi_{j k} / \ln 3
\end{array}\right.
$$

where $r_{j k}$ is the evaluation of expert $j$ linked to the $k^{\text {th }}$ performance index and $r_{j}$ and $H_{r j}$ are the total scores and entropy score for an expert. Then, the total entropy and the entropy weights of expert reliability can be set using:

$$
\left\{\begin{array}{c}
E_{e}=\sum_{j=1}^{M} H\left(r_{j}\right) \\
\theta_{j}=H\left(r_{j}\right) / E_{e}
\end{array}\right.
$$

Suppose $X_{j}$ is the credibility weight coefficient, which is assigned by taking into account the system familiarity, membership qualifications, and assessment environment. Correspondingly, the total credibility score of the expert, which is expressed as the normalized sum of the entropy and qualitative weights, is given by:

$$
h_{j}=\left(\theta_{j}+X j\right) /\left(\sum_{j=1}^{M}+X_{j}\right)
$$

The expert evaluations of the trapezoidal fuzzy number defined by three risk factors with regards to the failure mode are expressed as $S_{i j}=\left(S_{i j}^{L}, S_{i j}^{M_{1}}, S_{i j}^{M_{2}}, S_{i j}^{U}\right), O_{i j}=\left(O_{i j}^{L}, O_{i j}^{M_{1}}, O_{i j}^{M_{2}}, O_{i j}^{U}\right)$, respectively. As a result, the final fuzzy evaluations of risk factors related to the $i^{\text {th }}$ failure mode can be defined as:

$$
\left\{\begin{aligned}
& S_{i}=\sum_{j=1}^{M} h_{j} S_{i j}=\left(\sum_{j=1}^{M} h_{j} S_{i j}^{L}, \sum_{j=1}^{M} h_{j} S_{i j}^{M_{1}}, \sum_{j=1}^{M} h_{j} S_{i j}^{M_{2}}, \sum_{j=1}^{M} h_{j} S_{i j}^{U}\right) \\
& O_{i}=\sum_{j=1}^{M} h_{j} O_{i j}=\left(\sum_{j=1}^{M} h_{j} O_{i j}^{L}, \sum_{j=1}^{M} h_{j} O_{i j}^{M_{1}}, \sum_{j=1}^{M} h_{j} O_{i j}^{M_{2}}, \sum_{j=1}^{M} h_{j} O_{i j}^{U}\right) \\
& D_{i}=\sum_{j=1}^{M} h_{j} D_{i j}=\left(\sum_{j=1}^{M} h_{j} D_{i j}^{L}, \sum_{j=1}^{M} h_{j} D_{i j}^{M_{1}}, \sum_{j=1}^{M} h_{j} D_{i j}^{M_{2}}, \sum_{j=1}^{M} h_{j} D_{i j}^{U}\right)
\end{aligned}\right.
$$


Let $\omega_{S j}=\left(\omega_{S j}^{L}, \omega_{S j}^{M}, \omega_{S j}^{U}\right), \omega_{O j}=\left(\omega_{O j}^{L}, \omega_{O j^{\prime}}^{M} \omega_{O j}^{U}\right)$, and $\omega_{D j}=\left(\omega_{D j}^{L}, \omega_{D j}^{M}, \omega_{D j}^{U}\right)$ be triangular fuzzy values of the relative importance scores of three risk factors as done by an expert [28]. Next, the final fuzzy values of the risk factor weights can be expressed as:

$$
\left\{\begin{array}{l}
\omega_{S}=\sum_{j=1}^{M} h_{j} \omega_{S j}=\left(\sum_{j=1}^{M} h_{j} \omega_{S j}^{L}, \sum_{j=1}^{M} h_{j} \omega_{S j}^{M}, \sum_{j=1}^{M} h_{j} \omega_{S j}^{U}\right) \\
\omega_{O}=\sum_{j=1}^{M} h_{j} \omega_{O j}=\left(\sum_{j=1}^{M} h_{j} \omega_{O j^{\prime}}^{L}, \sum_{j=1}^{M} h_{j} \omega_{O j^{\prime}}^{M}, \sum_{j=1}^{M} h_{j} \omega_{O j}^{U}\right) \\
\omega_{D}=\sum_{j=1}^{M} h_{j} \omega_{D j}=\left(\sum_{j=1}^{M} h_{j} \omega_{D j}^{L}, \sum_{j=1}^{M} h_{j} \omega_{D j}^{M}, \sum_{j=1}^{M} h_{j} \omega_{D j}^{U}\right)
\end{array}\right.
$$

The fuzzy RPN is set as the fuzzy-evaluated geometric mean of three risk factors as follows:

$$
\exp \left[\sum_{\lambda=1}^{3}\left(\omega_{\lambda} \ln R_{\lambda}\right) / \sum_{\lambda=1}^{3} \omega_{\lambda}\right]
$$

Since $R_{\lambda}$ and $\omega_{\lambda}$ are fuzzy numbers $(\lambda=1,2,3)$, they cannot be calculated directly. The alpha-level set theory is adopted to compute the fuzzy number, which can be defined as:

$$
\begin{gathered}
F R P N=\sum_{\alpha}\left[(F R P N)_{\alpha}^{L},(F R P N)_{\alpha}^{U}\right] \\
\left\{\begin{array}{l}
(F R P N)_{\alpha}^{L}=\exp \left[\sum_{\lambda=1}^{3}\left(\left(\omega_{\lambda}\right)_{\alpha}^{L} \ln \left(R_{\lambda}\right)_{\alpha}^{L}\right) / \sum_{\lambda=1}^{3}\left(\omega_{\lambda}\right)_{\alpha}^{L}\right] \\
(F R P N)_{\alpha}^{U}=\exp \left[\sum_{\lambda=1}^{3}\left(\left(\omega_{\lambda}\right)_{\alpha}^{U} \ln \left(R_{\lambda}\right)_{\alpha}^{U}\right) / \sum_{\lambda=1}^{3}\left(\omega_{\lambda}\right)_{\alpha}^{U}\right]
\end{array}\right\}
\end{gathered}
$$

where $\alpha$ is the confidence level and $\left[(F R P N)_{\alpha}^{L},(F R P N)_{\alpha}^{U}\right]$ is the alpha-level set of the fuzzy $R P N$.

To perform the defuzzification of the fuzzy $R P N$, we adopted the centroid defuzzification, which is described as:

$$
X_{0}(F R P N)=\int x \cdot \mu_{F R P N}(x) d x / \int \mu_{F R P N}(x) d x
$$

where $x_{0}(F R P N)$ and $\mu_{F R P N}(x)$ are the defuzzified values and fuzzy membership functions of $R P N_{s}$. Here, we approximate the precise membership functions for MDF continuous not pressing. The information described by Equations (3)-(11) is to calculate the fuzzy RPN using fuzzy trigonometric functions and confidence weights. In this paper, the deviation level from the process quality control analysis of the MDF continuous hot pressing process and, finally, the deviation expert entropy and weight of the MDF continuous hot pressing process are expressed as the above Equation (3), where $r_{j k}$ is the expert $j$ score related to the performance index of the deviation level of the MDF hot pressing process, $r_{j}$ and $H_{r j}$ are the total score and entropy value in the expert system after the evaluation of this deviation level, and then, the total entropy value and the judgment of the deviation level result can be determined separately. The entropy weight is Equation (4). Finally, through the derivation process of the above equations, it is currently impossible to calculate the fuzzy number, so it is necessary to introduce the set theory; the confidence is the fuzzy RPN, and $\left[(F R P N)_{\alpha}^{L},(F R P N)_{\alpha}^{U}\right]$ the level set. The centroid deblurring number method is used to calculate the deviation level RPN value generated during the continuous hot pressing of MDF.

\section{Experiments and Analysis}

\subsection{RPN Compared with FRPN}

Through the calculation of the above algorithm equations, the calculated FRPN value corresponds to 20 kinds of MDF continuous hot pressure deviation levels, and the deviation level data in the 
continuous hot pressing process of MDF are calculated by the above-mentioned calculated fuzzy FMEA method, wherein FM indicates all the deviation levels after using the fuzzy FMEA method; the FRPN results are calculated according to the RPN values, and the final FRPN result is shown in Table 2.

The simulation results of FPRN calculated by the fuzzy FMEA method used in the third part applied to the continuous hot pressing of medium-density fiberboard are shown in Figure 4.

We show the construction of the simulation model of the decision system with Simulink 9.3 (part of MATLAB R2019a, MathWorks, Natick, MA, USA) in Figure 5. The model includes five detection channels. Each channel has a sensor for detecting the system output. They are separately denoted as S5, S4, S3, S2, and S1. The errors drive the sub-decision matrices through ECW. Moreover, the final decision matrix is the result of the logical operation $\mathrm{OR}(\mathrm{A}, \mathrm{B})$ of the sub-decision matrices. The procedure is achieved in nuclear synergy. The principle method is shown in Figure 5.

Table 2. The severity (S), degree of occurrence (O), and detectability (D) of the fuzzy scores of continuous hot pressing of MDF.

\begin{tabular}{lllllc}
\hline FM & (S) & (O) & (D) & RPN & FRPN \\
\hline FM1 & 2 & 4 & 6 & 48 & 25.2190 \\
FM2 & 4 & 4 & 6 & 96 & 60.3515 \\
FM3 & 6 & 8 & 6 & 288 & 230.7936 \\
FM4 & 4 & 2 & 6 & 48 & 25.2190 \\
FM5 & 8 & 4 & 6 & 192 & 160.3515 \\
FM6 & 2 & 4 & 6 & 48 & 25.2190 \\
FM7 & 3 & 4 & 6 & 72 & 35.6736 \\
FM8 & 6 & 4 & 6 & 144 & 100.6581 \\
FM9 & 6 & 6 & 4 & 144 & 100.6581 \\
FM10 & 8 & 6 & 6 & 288 & 230.7936 \\
FM11 & 2 & 4 & 6 & 48 & 25.2190 \\
FM12 & 3 & 5 & 6 & 90 & 55.1305 \\
FM13 & 4 & 4 & 6 & 96 & 60.3515 \\
FM14 & 6 & 4 & 6 & 144 & 100.6581 \\
FM15 & 8 & 4 & 6 & 192 & 132.7936 \\
FM16 & 2 & 4 & 6 & 48 & 25.2190 \\
FM17 & 3 & 6 & 6 & 108 & 75.8755 \\
FM18 & 4 & 4 & 6 & 96 & 60.3515 \\
FM19 & 6 & 6 & 4 & 144 & 100.6581 \\
FM20 & 8 & 6 & 6 & 288 & 230.7936 \\
\hline
\end{tabular}

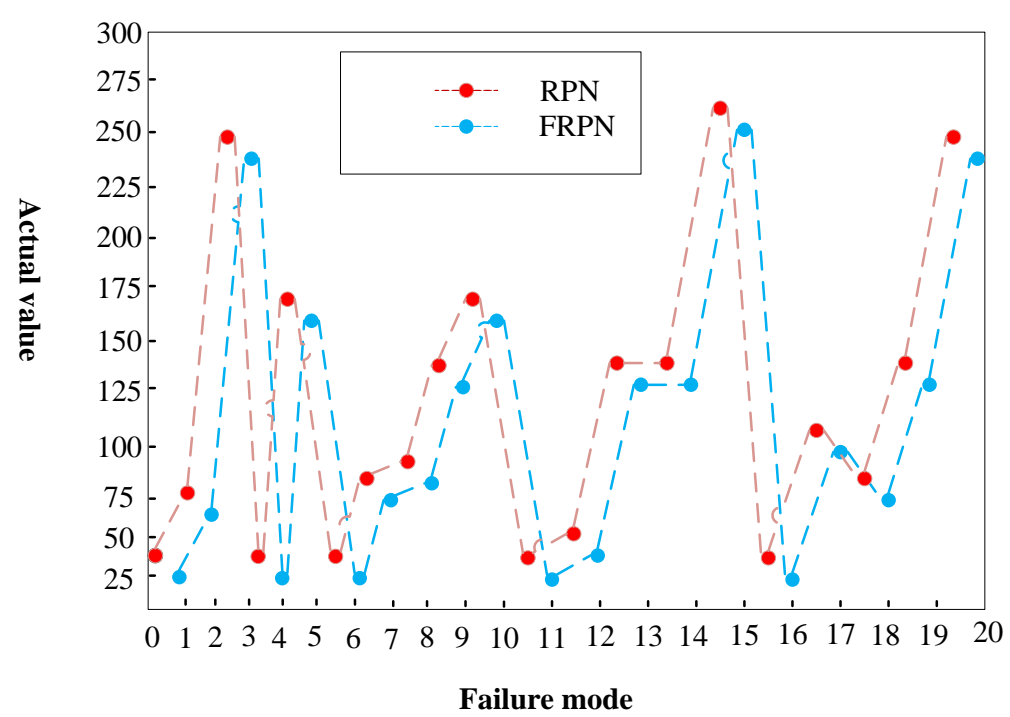

Figure 4. Numerical comparison of MDF continuous hot pressing process deviation grades, risk probability number (RPN) and fuzzy risk probability number (FRPN). 


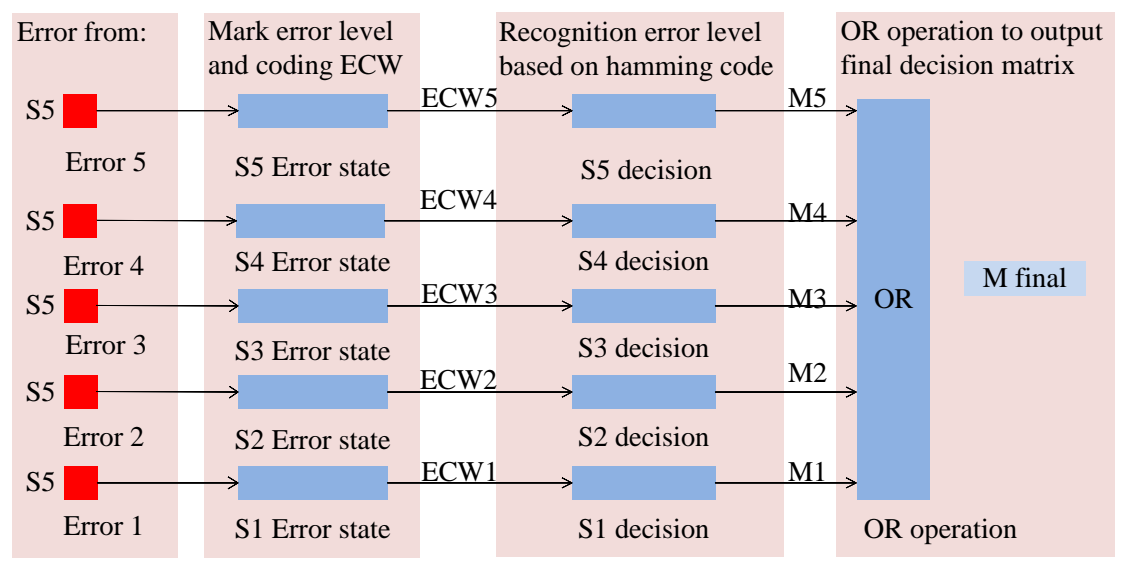

Figure 5. Decision model with three detection channels driven by error data. ECW-error correction word.

\subsection{ECW of MDF Continuous Hot Pressing}

Due to the similarity of the data in FRPN, the deviation mode values of the two modes or more are the same, and it is difficult to distinguish which specific mode is specified. The FRPN data results shown in Table 3 show that the FRPN values of FM8, FM9, FM14, and FM19 were the same and could not be determined as a specific fault mode. Therefore, the DS evidence theory was used to determine the values between zero and one for the unique sensor strategy values and RPN values, and the values were set by the expert group.

Table 3. The Dempster-Shafer (DS) evidence theory analysis of the fuzzy FMEA of MDF.

\begin{tabular}{ccl}
\hline Chwila & Sensor Strategy $\left(\boldsymbol{m}_{\mathbf{1}}\right)$ & $\mathbf{R P N}\left(\boldsymbol{m}_{\mathbf{2}}\right)$ \\
\hline FM8 & 0.80 & 0.20 \\
FM9 & 0.09 & 0.70 \\
FM14 & 0.01 & 0.05 \\
FM19 & 0.10 & 0.05 \\
\hline
\end{tabular}

In order to find the synthesis rule $\mathrm{m}(12)$, we first find the value of the normalization coefficient $1-K$.

$$
1-K=\sum_{B \cap C \neq \varphi} m_{1}(B) \cdot m_{2}(C)
$$

According to Equation (12), the value of $1-K$ in Table 3 can be calculated.

$$
\begin{aligned}
& 1-k=m_{1}(F M 8) \cdot m_{2}(F M 8)+m_{1}(F M 9) \\
& \cdot m_{2}(F M 9)+m_{1}(F M 14) \cdot m_{2}(F M 14) \\
& +m_{1}(F M 19) \cdot m_{2}(F M 19)
\end{aligned}
$$

The calculated value of $1-K$ is 0.2015 ; hence, we evaluate the mass function for each hypothesis after the composition.

(1) FM8's combined function value of the mass: $m_{1} \oplus m_{2}(\{F M 8\})=\frac{1}{1-K} \sum_{B \cap C=\{F M 8\}} m_{1}(B)$. $m_{2}(C)=\frac{1}{1-K} \cdot m_{1}(\{F M 8\}) \cdot m_{2}(\{F M 8\})$ The calculation result is 0.394 .

(2) FM9's combined function value of the mass: $m_{1} \oplus m_{2}(\{F M 9\})=\frac{1}{1-K} \sum_{B \cap C=\{F M 9\}} m_{1}(B)$. $m_{2}(C)=\frac{1}{1-K} \cdot m_{1}(\{F M 9\}) \cdot m_{2}(\{F M 9\})$ The calculation result is 0.313 .

(3) FM14's combined function value of the mass: $m_{1} \oplus m_{2}(\{F M 14\})=\frac{1}{1-K} \sum_{B \cap C=\{F M 14\}} m_{1}(B)$. $m_{2}(C)=\frac{1}{1-K} \cdot m_{1}(\{F M 14\}) \cdot m_{2}(\{F M 14\})$ The calculation result is 0.00248 . 
(4) FM19's combined function value of the mass: $m_{1} \oplus m_{2}(\{F M 19\})=\frac{1}{1-K} \sum_{B \cap C=\{F M 19\}} m_{1}(B)$. $m_{2}(C)=\frac{1}{1-K} \cdot m_{1}(\{F M 19\}) \cdot m_{2}(\{F M 19\})$ The calculation result is 0.2481 . According to the normalization of FM8, FM9, FM14, and FM1, the mode of this deviation level is FM9.

\subsection{MDF Hamming Coding}

The deviation information of the MDF sheet is calculated by using the Hamming coding method. For sensor $S_{s}$, code the error level bits string $B_{e s}$ into ECW $W_{s}$. The bit numbers of $B_{e s}$ and $W_{s}$ are separately $N_{B s}$ and $W_{s}$. They should satisfy Equation (13). It is also more than $N_{W s}$. Standard $B_{0}$ is constant. Actual error level string $B_{e s}$ is variable and reflects the real error condition, but is not transferred. Therefore, it is not necessary to receive an $N_{B s}+N_{W s}$ bit Hamming code for nuclear synergy. ECW $N_{W s}$ with bits is enough for the decision. It is learned from Equation (13) that transferring the object is ECW $N_{W s}$ with bits, but the number of error levels $N_{B s} N_{W s}$ does not grow with $N_{B s}$ in a logarithmic function, e.g., not linear. It is shown in Figure 6a, within a certain limit, that employing ECW to represent error data permits the increase of the error levels' number. It makes the optimization of the controller more convenient. More detection channels are appended to the increasing tendencies of $N_{B s}$ and $N_{W s}$ with $S_{s}$. The usage of ECW reduces the bit numbers of representation and operation. It is beneficial for not only saving hardware resources to transfer and calculate, but also improves efficiency in recognition. Obviously, in any case, to develop control performance, refining error levels or appending an efficient operation flocking system, the number of sensors increases. For $S_{s}$ and also satisfying Equation (13). Figure $6 \mathrm{~b}$ shows the error from the five sensors.

$$
N_{B s}+N_{W s}+1 \leq 2^{N_{W s}}
$$
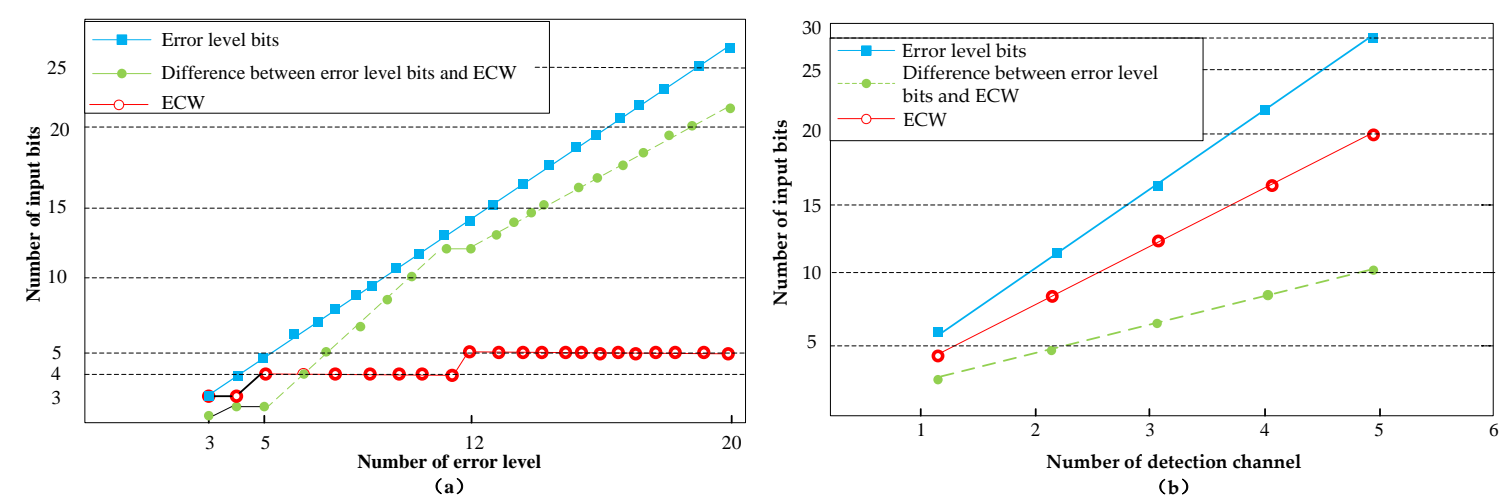

Figure 6. (a) Comparison of the number of error levels and coded error data in the channel. (b) Increasing tendencies for various modes.

\subsection{Self-Coordination and Mutual Cooperation of the MDF Continuous Hot Press Cylinder}

The set of reference inputs for three detection channels are sine signals. These signals mean that errors are time-variable. Their frequency, amplitude, and bias were separately $1.256,0.5$, and 0.5 . The phases were different and set separately as $0,1.775$, and 7.85 . The input error signals constructed are shown in Figure 7. The errors should be zero, as the output of the terminals is set to one. At most sampling points, errors from different channels belong to different error levels. This should generate identifiable ECWs and drive relevant sub-decision matrices. 


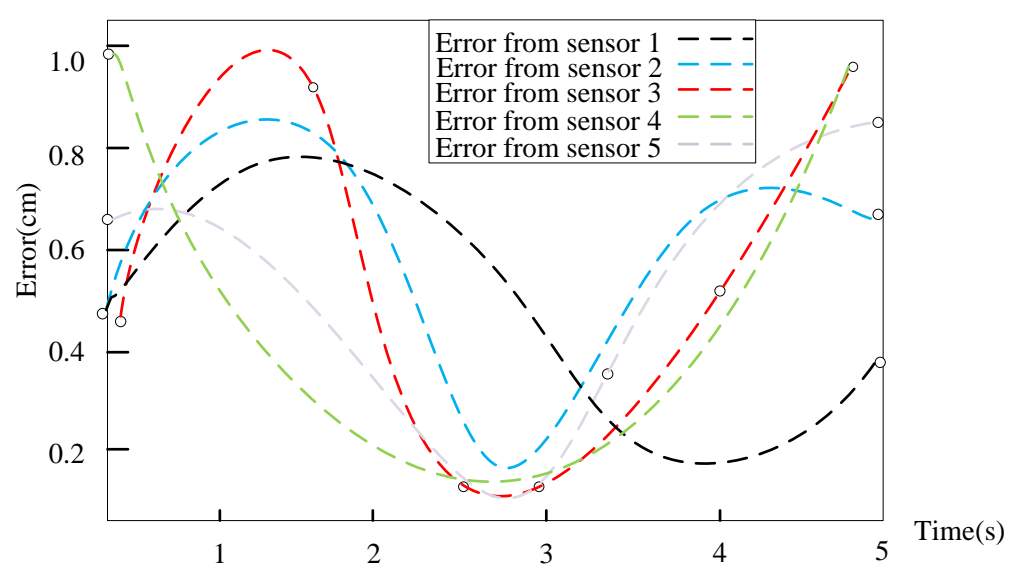

Figure 7. Reference inputs of channels with sensor 1 (S1), S2, S3, S4, and S5.

The synergy relation of the flocking system is shown in Figure 8. Terminals in a common ellipse compose a synergy unit. They are self-synergetic if their output modes are the same, otherwise they are inter-synergetic. Furthermore, in a common unit, the displacement of the terminal outputs is always the primary one. Terminals marked 23, 33, and 43 always act in Mode 1, which means they output displacement. Terminals act in Mode 0 to output pressure.

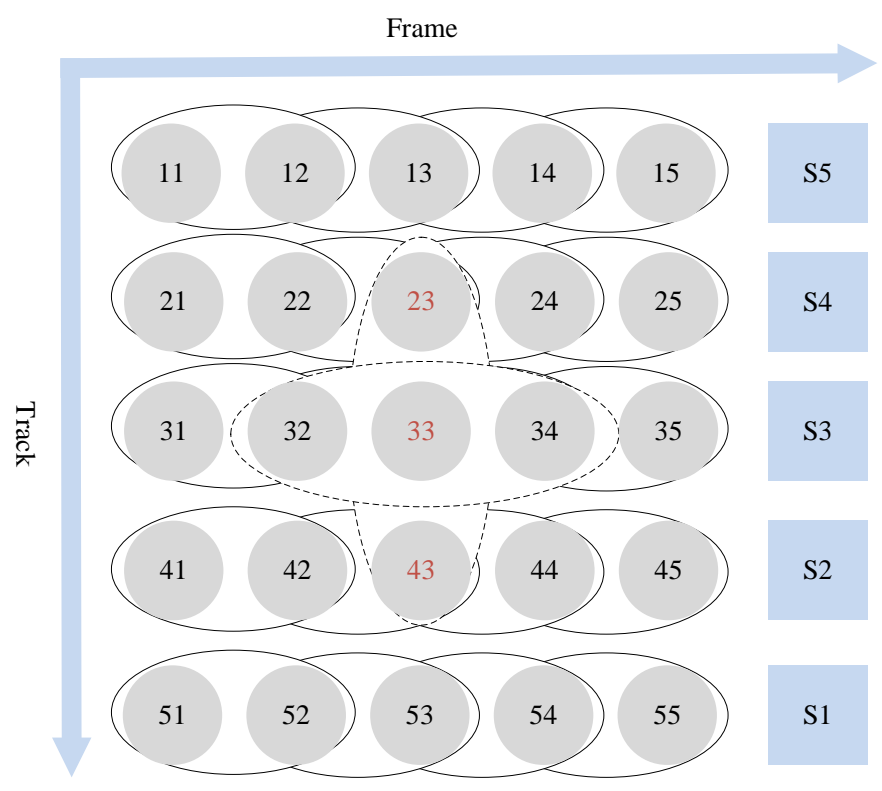

Figure 8. Hierarchical synergy corrections.

In Figure 9, we set the thickness of the load to $9 \mathrm{~mm}$. The experimental load was conveyed to pass the thickness detection sensors. $T_{\text {test }}$ represents the thickness test value of S1 to S5. $E_{\text {act }}$ represents the error range generated by the five deviation levels. Error correcting word (ECW) represents the deviation Hamming code analysis result of the five deviation levels. The sensors collect actual thickness values and submit the data to calculate errors. The collected data are listed in Equation (14). Actual errors are calculated according to Equation (15). The actual errors from Sensors 5 to 1 are separately shown for Level 5, Level 4, Level 3, Level 2, and Level 1. The ECW is as Equation (16) and Figure 10. 


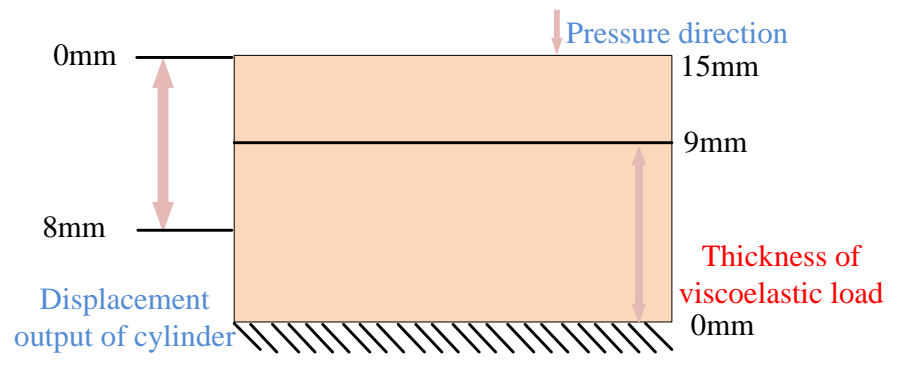

Figure 9. Thickness of the viscoelastic load and output of the cylinder.

$$
\begin{aligned}
& \left\{\begin{array}{l}
T_{\text {test_S5 }}=9.5 \mathrm{~mm} \\
T_{\text {test_S4 }}=9.4 \mathrm{~mm} \\
T_{\text {test_S3 }}=9.3 \mathrm{~mm} \\
T_{\text {test_S2 }}=9.2 \mathrm{~mm} \\
T_{\text {test_S1 }}=9.1 \mathrm{~mm}
\end{array}\right. \\
& \left\{\begin{array}{l}
E_{\text {act_S5 }}=\frac{\left(T_{\text {test_S5 }}-T_{\text {set }}\right)}{T_{\text {set }}}=5.56 \% \Rightarrow V<E_{\text {act_S5 }}<I V \\
E_{\text {act_S4 }}=\frac{\left(T_{\text {test_S4 }}-T_{\text {set }}\right)}{T_{\text {set }}}=5.13 \% \Rightarrow I V<E_{\text {act_S4 }}<I I I \\
E_{\text {act_S3 }}=\frac{\left(T_{\text {test_S3 }}-T_{\text {set }}\right)}{T_{\text {set }}}=4.41 \% \Rightarrow I I I<E_{\text {act_S3 }}<I I \\
E_{\text {act_S2 }}=\frac{\left(T_{\text {test_S2 }}-T_{\text {set }}\right)}{T_{\text {set }}}=3.82 \% \Rightarrow I I<E_{\text {act_S2 }}<I \\
E_{\text {act_S1 }}=\frac{\left(T_{\text {test_S1 }}-T_{\text {set }}\right)}{T_{\text {set }}}=3.34 \% \Rightarrow 0<E_{\text {act_S1 }}<I
\end{array}\right. \\
& \left\{\begin{array}{l}
E C W_{5}=0110 \\
E C W_{4}=0010 \\
E C W_{3}=1010 \\
E C W_{2}=1011 \\
E C W_{1}=1010
\end{array}\right.
\end{aligned}
$$

The units are separately presented in $\mathrm{mm}$ and bar. The output surfaces are shown in Figure 10. Errors from the sensors were eliminated. Pressure output values with the same displacement output were different so as to have the displacement outputs of flocking terminals present favorable consistency because viscoelasticity was unevenly presented at the surface of the load in Figure 11.

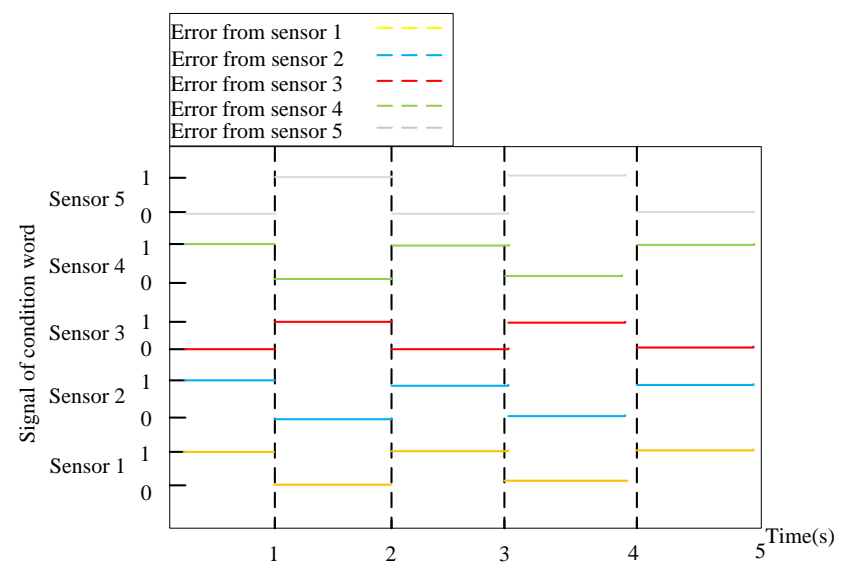

Figure 10. ECWs coded as system errors. 


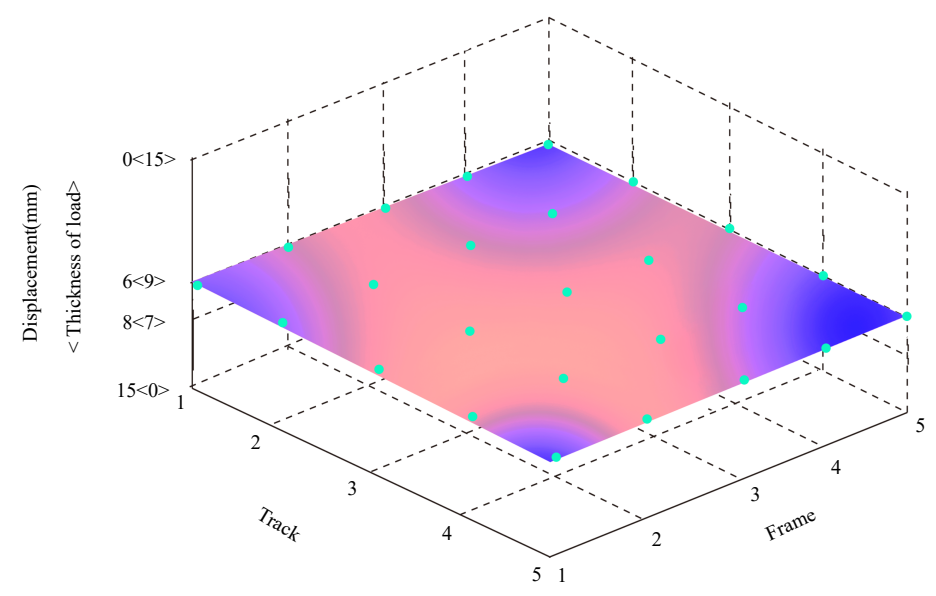

Figure 11. Pressure output surface of flocking terminals.

As the results showed, the 20 kinds of deviation levels were divided into four types, and each type was divided into five deviation levels, namely A, B, C, D, and E. There were two types of slopes: left side slope and right side slope. Corresponding to different level correction methods respectively, the RPN value of the left mild slope A level was 48, the OR operation calculation result was $M_{01}$, and the nearest value could be judged as the deviation type of FM4. The RPN value of the left mild slope B level was 96; the OR operation calculation result was $M_{02}$; the nearest value could be judged as the deviation type of FM4. The RPN value of the left moderate slope C level was 288; the OR operation calculation result was $M_{03}$; the nearest value could be judged as the deviation type of FM1. The RPN value of the left heavy slope D level was 48 ; the OR operation calculation result was $M_{04}$; the nearest value could be judged as the deviation type of FM3. The RPN value of the right heavy slope E level was 48 ; the OR operation calculation result was $M_{05}$; the nearest value could be judged as the deviation type of FM3.

The RPN value of the right mild slope A level was 48; the OR operation calculation result was $M_{06}$; the nearest value could be judged as the deviation type of FM6. The RPN value of the right mild slope B level was 72; the OR operation calculation result was $M_{07}$; the nearest value could be judged as the deviation type of FM6. The RPN value of the right moderate slope C level was 144; the OR operation calculation result was $M_{08}$; the nearest value could be judged as the deviation type of FM9. The RPN value of the right heavy slope D level was 144; the OR operation calculation result was $M_{09}$; the nearest value could be judged as the deviation type of FM7. The RPN value of the right heavy slope E level was 288; the OR operation calculation result was $M_{10}$; the nearest value could be judged as the deviation type of FM7.

The RPN value of the light depression A level was 48; the OR operation calculation result was $M_{11}$; the nearest value could be judged as the deviation type of FM20. The RPN value of the light depression B level was 108; the OR operation calculation result was $M_{12}$; the nearest value could be judged as the deviation type of FM20. The RPN value of the moderate depression C level was 96; the OR operation calculation result was $M_{13}$; the nearest value could be judged as the deviation type of FM18. The RPN value of the heavy depression D level was 144; the OR operation calculation result was $M_{14}$; the nearest value could be judged as the deviation type of FM17. The RPN value of the heavy depression E level was 288; the OR operation calculation result was $M_{15}$; the nearest value could be judged as the deviation type of FM17.

The RPN value of the mild bulge A level was 48; the operation calculation result was $M_{16}$; the nearest value could be judged as the deviation type of FM12. The RPN value of the mild bulge B level was 90; the operation calculation result was $M_{17}$; the nearest value could be judged as the deviation type of FM12. The RPN value of the moderate bulge $C$ level was 96; the operation calculation result was $M_{18}$; the nearest value could be judged as the deviation type of FM13. The RPN value of 
the heavy bulge D level was 144; the operation calculation result was $M_{19}$; the nearest value could be judged as the deviation type of FM14. The RPN value of the heavy bulge E level was 192; the operation calculation result was $M_{20}$; the nearest value could be judged as the deviation type of FM14.

$$
\begin{aligned}
& M_{01}=\left[\begin{array}{lllll}
0 & 1 & 0 & 0 & 1 \\
0 & 1 & 1 & 0 & 0 \\
0 & 1 & 0 & 0 & 0 \\
0 & 1 & 0 & 0 & 0 \\
0 & 1 & 0 & 0 & 0
\end{array}\right] M_{02}=\left[\begin{array}{lllll}
0 & 1 & 0 & 0 & 1 \\
0 & 1 & 1 & 0 & 0 \\
0 & 1 & 0 & 0 & 0 \\
0 & 1 & 0 & 0 & 0 \\
0 & 1 & 0 & 0 & 0
\end{array}\right] \\
& M_{03}=\left[\begin{array}{lllll}
0 & 1 & 1 & 0 & 1 \\
0 & 1 & 1 & 0 & 1 \\
0 & 1 & 0 & 0 & 0 \\
0 & 1 & 0 & 0 & 0 \\
0 & 1 & 0 & 0 & 0
\end{array}\right] M_{04}=\left[\begin{array}{lllll}
0 & 1 & 0 & 0 & 1 \\
0 & 1 & 1 & 0 & 1 \\
0 & 1 & 0 & 0 & 1 \\
0 & 1 & 0 & 0 & 1 \\
0 & 1 & 0 & 0 & 0
\end{array}\right] \\
& M_{05}=\left[\begin{array}{lllll}
0 & 1 & 0 & 0 & 1 \\
0 & 1 & 1 & 0 & 1 \\
0 & 1 & 0 & 0 & 1 \\
0 & 1 & 0 & 0 & 1 \\
0 & 1 & 0 & 0 & 0
\end{array}\right] M_{06}=\left[\begin{array}{lllll}
1 & 1 & 0 & 0 & 0 \\
0 & 1 & 1 & 0 & 0 \\
0 & 1 & 0 & 0 & 0 \\
0 & 1 & 0 & 0 & 0 \\
0 & 1 & 0 & 0 & 0
\end{array}\right] \\
& M_{07}=\left[\begin{array}{lllll}
1 & 1 & 0 & 0 & 0 \\
0 & 1 & 1 & 0 & 0 \\
0 & 1 & 0 & 0 & 0 \\
0 & 1 & 0 & 0 & 0 \\
0 & 1 & 0 & 0 & 0
\end{array}\right] M_{08}=\left[\begin{array}{lllll}
1 & 1 & 0 & 0 & 0 \\
1 & 1 & 1 & 0 & 0 \\
1 & 1 & 0 & 0 & 0 \\
0 & 1 & 0 & 0 & 0 \\
0 & 1 & 0 & 0 & 0
\end{array}\right] \\
& M_{09}=\left[\begin{array}{lllll}
1 & 1 & 0 & 0 & 0 \\
1 & 1 & 1 & 0 & 0 \\
1 & 1 & 0 & 0 & 0 \\
0 & 1 & 0 & 0 & 0 \\
0 & 1 & 0 & 0 & 0
\end{array}\right] M_{10}=\left[\begin{array}{lllll}
0 & 1 & 0 & 0 & 0 \\
0 & 1 & 1 & 0 & 0 \\
0 & 1 & 0 & 0 & 0 \\
0 & 1 & 0 & 0 & 0 \\
0 & 1 & 0 & 0 & 0
\end{array}\right] \\
& M_{11}=\left[\begin{array}{lllll}
0 & 1 & 0 & 0 & 0 \\
0 & 1 & 1 & 0 & 0 \\
1 & 1 & 1 & 0 & 0 \\
0 & 1 & 0 & 0 & 0 \\
0 & 1 & 0 & 0 & 0
\end{array}\right] \quad M_{12}=\left[\begin{array}{lllll}
0 & 1 & 1 & 0 & 0 \\
0 & 1 & 0 & 0 & 0 \\
1 & 1 & 0 & 0 & 0 \\
1 & 1 & 0 & 0 & 0 \\
0 & 1 & 0 & 0 & 0
\end{array}\right] \\
& M_{13}=\left[\begin{array}{lllll}
0 & 1 & 0 & 0 & 0 \\
0 & 1 & 1 & 1 & 0 \\
0 & 1 & 1 & 0 & 0 \\
0 & 1 & 0 & 0 & 0 \\
0 & 1 & 0 & 0 & 0
\end{array}\right] M_{14}=\left[\begin{array}{lllll}
0 & 1 & 0 & 0 & 0 \\
0 & 1 & 1 & 1 & 0 \\
0 & 1 & 1 & 0 & 0 \\
0 & 1 & 0 & 0 & 0 \\
0 & 1 & 0 & 0 & 0
\end{array}\right] \\
& M_{15}=\left[\begin{array}{lllll}
0 & 1 & 0 & 0 & 0 \\
0 & 1 & 1 & 1 & 0 \\
0 & 1 & 1 & 1 & 0 \\
0 & 1 & 0 & 0 & 0 \\
0 & 1 & 0 & 0 & 0
\end{array}\right] M_{16}=\left[\begin{array}{lllll}
0 & 1 & 0 & 0 & 0 \\
0 & 1 & 1 & 1 & 0 \\
0 & 1 & 1 & 1 & 0 \\
0 & 1 & 0 & 0 & 0 \\
0 & 1 & 0 & 0 & 0
\end{array}\right] \\
& M_{17}=\left[\begin{array}{lllll}
0 & 1 & 0 & 0 & 0 \\
0 & 1 & 1 & 1 & 0 \\
0 & 1 & 1 & 1 & 0 \\
0 & 1 & 0 & 0 & 0 \\
0 & 1 & 0 & 0 & 0
\end{array}\right] M_{18}=\left[\begin{array}{lllll}
0 & 1 & 0 & 0 & 0 \\
0 & 1 & 1 & 1 & 0 \\
0 & 1 & 1 & 0 & 0 \\
0 & 1 & 0 & 0 & 0 \\
0 & 1 & 0 & 0 & 0
\end{array}\right]
\end{aligned}
$$




$$
M_{19}=\left[\begin{array}{lllll}
0 & 1 & 0 & 0 & 0 \\
0 & 1 & 1 & 1 & 0 \\
0 & 1 & 1 & 0 & 0 \\
0 & 1 & 0 & 0 & 0 \\
0 & 1 & 0 & 0 & 0
\end{array}\right] M_{20}=\left[\begin{array}{lllll}
0 & 1 & 1 & 1 & 0 \\
0 & 1 & 1 & 0 & 0 \\
0 & 1 & 1 & 0 & 0 \\
0 & 1 & 0 & 0 & 0 \\
0 & 1 & 0 & 0 & 0
\end{array}\right]
$$

\section{Conclusions}

We proposed a fuzzy failure mode and effects analysis (FMEA) method, which combined fault theory and a failure analysis method. The method addressed the problems of board thickness control failure and thickness deviation defect blanking, which could occur during the continuous hot pressing process during the production of medium-density fiberboard (MDF). The method combined the fault analysis with the Hamming code method in order to calculate and represent the cylinder array of the continuous hot pressed thickness control execution unit to analyze and to process the potential fixed thickness failure modes in continuous hot pressing during the production of MDF and then summarized the decision rules for controlling the board thickness and the level of sheet deviation. By combining the fuzzy FMEA method of the Hamming code and the logical OR operation of the experimental analysis, the proposed method to represent error data permitted the increase of the number of error levels, which made the optimization of the controller more convenient. This was beneficial for not only saving hardware resources to transfer and calculate, but also for improving the efficiency to recognize errors.

Author Contributions: Y.L. (Yunlei Lv) comprehensively studied the deviation and recognition of medium-density fiberboard in the fuzzy FMEA algorithm and the verification of this method, described the research findings, and wrote manuscripts, Y.L. (Yaqiu Liu) and W.J. provided guidance and experimental equipment in this paper. Writing, review and editing, M.W., R.S., and R.D.; supervision, W.W.; funding acquisition, W.J. All authors read and agreed to the published version of the manuscript.

Funding: This work is supported by the Key Research and Development Program of Shaanxi Province (No. 2018ZDXM-GY-036) and Shaanxi Key Laboratory of Intelligent Processing for Big Energy Data (No. IPBED7).

Conflicts of Interest: The authors declare no conflict of interest. The funders had no role in the design of the study; in the collection, analyses, or interpretation of data; in the writing of the manuscript; nor in the decision to publish the results.

\section{Abbreviations}

The following abbreviations are used in this manuscript:

$\begin{array}{ll}\text { CHP } & \text { continuous hot pressing } \\ \text { DS } & \text { Dempster-Shafer } \\ \text { FMEA } & \text { failure mode and effects analysis } \\ \text { FRPN } & \text { fuzzy risk priority number } \\ \text { IT2FS } & \text { interval type-2 fuzzy sets } \\ \text { MDF } & \text { medium-density fiberboard } \\ \text { RPN } & \text { risk probability number }\end{array}$

\section{References}

1. Akbulut, T.; Ayrilmis, N. Some advantages of three-layer medium-density fibreboard as compared to the traditional single-layer one. J. Wood Sci. 2019, 65, 46. [CrossRef]

2. Ates, S.; Kara, H.R.; Olgun, C.; Ozkan, O.E. Effects of heat treatment on some properties of MDF (medium-density fiberboard). Wood Mater. Sci. Eng. 2017, 12, 158-164. [CrossRef]

3. Euring, M.; Kirsch, A.; Kharazipour, A. Pre-pressing and pre-heating via hot-air/hot-steam process for the production of binderless medium-density fiberboards. BioResources 2016, 11, 6613-6624. [CrossRef]

4. Wu, Q. Synergetic Control for Slab Thickness Deviation Rectification of MDF Continuous Press. Ph.D. Thesis, Northeast Forestry University, Harbin, China, 2013. (In Chinese) 
5. Gul, W.; Khan, A.; Shakoor, A. Impact of hot pressing temperature on medium density fiberboard (MDF) performance. Adv. Mater. Sci. Eng. 2017, 2017, 1-6. [CrossRef]

6. Zhu, L.; Wang, Z.; Qiang, H.; Liu, Y. Global sliding-mode dynamic surface control for MDF continuous hot pressing slab thickness via LESO. Int. J. Mach. Learn. Cybern. 2019, 10, 1249-1258. [CrossRef]

7. Zhu, L.; Wang, Z.; Zhou, Y.; Liu, Y. Adaptive neural network saturated control for MDF continuous hot pressing hydraulic system with uncertainties. IEEE Access 2017, 6, 2266-2273. [CrossRef]

8. Zhu, L.; Zhou, Y.; Liu, Y. Robust adaptive neural prescribed performance control for MDF continuous hot pressing system with input saturation. IEEE Access 2018, 6, 9099-9113. [CrossRef]

9. Zhu, L.; Zhou, Y.; Wang, Z.; Liu, Y. A novel control for MDF continuous hot pressing accurate tracking: Adaptive fuzzy approach. Int. J. Comput. Appl. Technol. 2019, 59, 175-184. [CrossRef]

10. Lv, B.; Liu, Y.Q.; Zhu, L.K. Error-driven decision and error condition word for continuous hot press flocking control system. Int. J. Model. Identif. Control 2016, 26, 324-335.

11. Urbonas, A.; Raudonis, V.; Maskeliunas, R.; Damaševičius, R. Automated identification of wood veneer surface defects using faster region-based convolutional neural network with data augmentation and transfer learning. Appl. Sci. 2019, 9, 4898. [CrossRef]

12. Juočas, L.; Raudonis, V.; Maskeliūnas, R.; Damaševičius, R.; Woźniak, M. Multi-focusing algorithm for microscopy imagery in assembly line using low-cost camera. Int. J. Adv. Manuf. Technol. 2019, 102, 3217-3227. [CrossRef]

13. Huang, J.; You, J.-X.; Liu, H.-C.; Song, M.-S. Failure mode and effect analysis improvement: A systematic literature review and future research agenda. Reliab. Eng. Syst. Saf. 2020, 199, 106885. [CrossRef]

14. Sho, Y.; Hidenori, K.; Keiji, S. Extended Flocking Algorithm for Self-parameter Tuning. Electron. Commun. Jpn. 2015, 98, 44-51.

15. Nie, W.B.; Liu, W.D.; Chen, B.S. Application of Fuzzy Matrix Correlation and Risk Preference in FMEA Decision Making. Stat. Decis. Mak. 2019, 35, 54-58. (In Chinese)

16. Wu, Q.; Lv, B.; Wang, L.; Bai, J. Hamming Code Specification Analysis Based on Binary Tree Structure. Highlights Sci. 2015, 8, 540-548.

17. Qin, J.; Xi, Y.; Pedrycz, W. Failure mode and effects analysis (FMEA) for risk assessment based on interval type-2 fuzzy evidential reasoning method. Appl. Soft Comput. J. 2020, 89, 106134. [CrossRef]

18. Akyuz, C. A quantitative risk analysis by using interval type-2 fuzzy FMEA approach: The case of oil spill. Marit. Policy Manag. 2018, 45, 8. [CrossRef]

19. Dong, G.; Wei, W.; Xia, X.; Woźniak, M.; Damaševičius, R. Safety risk assessment of a Pb-Zn mine based on fuzzy-grey correlation analysis. Electronics 2020, 9, 130. [CrossRef]

20. Wei, W.; Xia, X.; Wozniak, M.; Fan, X.; Damaševičius, R.; Li, Y. Multi-sink distributed power control algorithm for Cyber-physical-systems in coal mine tunnels. Comput. Netw. 2019, 161, 210-219. [CrossRef]

21. Seiti, H.; Hafezalkotob, A.; Najafi, S.E.; Khalaj, M. A risk-based fuzzy evidential framework for FMEA analysis under uncertainty: An interval-valued DS approach. J. Intell. Fuzzy Syst. 2018, 35, 2. [CrossRef]

22. Liu, S.G.; Zhang, X.J.; Lan, Y. An Improved Assessment Method for FMEA for a Shipboard Integrated Electric Propulsion System Using Fuzzy Logic and DEMATEL Theory. J. Energy 2019, 12, 3162. [CrossRef]

23. McElroy, L.M.; Khorzad, R.; Nannicelli, A.P.; Brown, A.R.; Ladner, D.P.; Holl, J.L. Failure mode and effects analysis: A comparison of two common risk prioritisation methods. BMJ Qual. Saf. 2015, 25, 329-336. [CrossRef] [PubMed]

24. Fattahi, R.; Khalilzadeh, M. Risk evaluation using a novel hybrid method based on FMEA, extended MULTIMOORA, and AHP methods under fuzzy environment. Saf. Sci. 2018, 102, 290-300. [CrossRef]

25. Li, Z.; Chen, L. A novel evidential FMEA method by integrating fuzzy belief structure and grey relational projection method. Eng. Appl. Artif. Intell. 2019, 77, 136-147. [CrossRef]

26. Shafiee, M.; Enjema, E.; Kolios, A. An integrated FTA-FMEA model for risk analysis of engineering systems: A case study of subsea blowout preventers. Appl. Sci. 2019, 9, 1192. [CrossRef]

27. Fang, H.; Li, J.; Song, W. Failure mode and effects analysis: An integrated approach based on rough set theory and prospect theory. Soft Comput. 2020, 24, 6673-6685. [CrossRef]

28. Chen, L.; Deng, Y. A new failure mode and effects analysis model using Dempster-Shafer evidence theory and grey relational projection method. Eng. Appl. Artif. Intell. 2018, 76, 13-20. [CrossRef]

29. Tooranloo, H.S.; Ayatollah, A.S.; Alboghobish, S. Evaluating knowledge management failure factors using intuitionistic fuzzy FMEA approach. Knowl. Inf. Syst. 2018, 57, 183-205. [CrossRef] 
30. Arabsheybani, A.; Paydar, P.M.; Safaei, A.S. An integrated fuzzy MOORA method and FMEA technique for sustainable supplier selection considering quantity discounts and supplier's risk. J. Clean. Prod. 2018, 190, 577-591. [CrossRef]

31. An, X.; Cai, W.G.; Song, X.J. FMEA coupling evaluation method based on cloud model and collaborative decision. Comput. Integr. Manuf. Syst. 2018, 24, 1179-1190. [CrossRef]

32. Zh, S.L.; Su, X.L. Evaluation of water resources allocation plan in the Yellow River Basin coupled with game theory and DS evidence theory. J. Northwest A F Univ. (Nat. Sci. Ed.) 2019, 47, 123-133. (In Chinese)

33. Martin, R.; Zhang, J.; Liu, C. Dempster-Shafer Theory and Statistical Inference with Weak Beliefs. J. Stat. Sci. 2010, 1, 72-87. [CrossRef]

34. Liu, Y.Q.; Wu, Q. Slab Thickness Deviation Rectification Control of MDF Continuous Press Based on Hierarchical Structure of Synergetic Governance. In Proceedings of the International Conference on Mechatronics and Control, Jinzhou, China, 3-5 July 2014; pp. 855-860.

35. Nie, W.B.; Liu, W.D.; Hu, K.; Chen, H.; Zheng, T. Process failure risk assessment based on group decision and generalized Hausdorff distance. Comput. Integr. Manuf. Syst. 2015, 21, 2484-2493. (In Chinese)

36. Nie, W.B.; Liu, W.D.; Wu, Z.Y.; Chen, B.S.; Wu, L.L. Failure mode and effects analysis by integrating Bayesian fuzzy assessment number and extended gray relational analysis-technique for order preference by similarity to ideal solution method. Qual. Reliab. Eng. Int. 2019, 35, 1676-1697. [CrossRef]

37. Wu, H.; Lin, Y. Risk assessment for a floating attitude tension leg platform by application of a hybrid fuzzy-statistical process control model. J. Zhejiang Univ. Sci. A 2019, 20, 515-532. [CrossRef]

(C) 2020 by the authors. Licensee MDPI, Basel, Switzerland. This article is an open access article distributed under the terms and conditions of the Creative Commons Attribution (CC BY) license (http:/ / creativecommons.org/licenses/by/4.0/). 